\title{
A NOTE ON EQUILIBRIUM POINTS OF GREEN'S FUNCTION
}

\author{
ALEXANDER YU. SOLYNIN
}

(Communicated by Juha M. Heinonen)

\begin{abstract}
We answer a question raised by Ahmet Sebbar and Thérèse Falliero (2007) by showing that for every finitely connected planar domain $\Omega$ there exists a compact subset $K \subset \Omega$, independent of $w$, containing all critical points of Green's function $G(z, w)$ of $\Omega$ with pole at $w \in \Omega$.
\end{abstract}

Let $\Omega$ be a domain on $\mathbb{C}$ bordered by $n \geq 2$ Jordan analytic curves and let $G(z, w)$ be Green's function of $\Omega$ with pole at $w \in \Omega$. For $w \in \Omega$, let $\mathbb{Z}_{\Omega}(w)=\{z \in$ $\left.\Omega: \frac{\partial}{\partial z} G(z, w)=0\right\}$ be the set of critical (= equilibrium) points of $G(z, w)$. It is well known that for every $w \in \Omega$, \# $\left(\mathbb{Z}_{\Omega}(w)\right)=n-1$ counting multiplicity.

Theorem 1. Let $\overline{\mathbb{Z}}_{\Omega}$ denote the closure of the set $\mathbb{Z}_{\Omega}=\bigcup_{w \in \Omega} \mathbb{Z}_{\Omega}(w)$. Then $\overline{\mathbb{Z}}_{\Omega}$ is a compact subset of $\Omega$ having at most $n-1$ connected components.

Thus this theorem answers affirmatively a question raised by Ahmet Sebbar and Thérèse Falliero in $[2$, p. 314]. For the case when $\Omega$ is doubly-connected, this theorem was proved in [2] by direct computation involving an explicit expression of Green's function of a circular annulus.

To prove Theorem 1, we use the well-known Schiffer's formula linking Green's function with the Bergman kernel. The Bergman kernel and the adjoint Bergman kernel of $\Omega$ are defined as $K(z, w)=-\frac{2}{\pi} \frac{\partial^{2}}{\partial z \partial \bar{w}} G(z, w)$ and $L(z, w)=-\frac{2}{\pi} \frac{\partial^{2}}{\partial z \partial w} G(z, w)$, respectively. Necessary properties of the Bergman kernel can be found, for example, in [1]. It is convenient to use subscripts to denote differentiation so that $G_{z}(z, w)=\frac{\partial}{\partial z} G(z, w), G_{z \bar{w}}(z, w)=\frac{\partial^{2}}{\partial z \partial \bar{w}} G(z, w)$, etc. To emphasize dependence on $\Omega$ if necessary, we will write $G_{\Omega}(z, w), K_{\Omega}(z, w)$, etc.

Lemma 1. Let $\Omega$ be a Dirichlet domain in the upper half-plane $\mathbb{H}=\{z: \Im z>0\}$ having an open interval $I=(-1,1)$ on $\partial \Omega$ and such that $\widehat{\Omega}=\Omega \cup \Omega^{*} \cup I$ is a domain. Here $\Omega^{*}=\{z: \bar{z} \in \Omega\}$. Then

$$
K_{\Omega}(z, w)=K_{\widehat{\Omega}}(z, w)-L_{\widehat{\Omega}}(z, \bar{w}), \quad z \in \Omega, w \in \Omega \cup I, z \neq w,
$$

and

$$
\lim _{\mathbb{H} \ni w \rightarrow 0} \frac{\frac{\partial}{\partial z} G_{\Omega}(z, w)}{\Im w}=\pi i K_{\Omega}(z, 0)
$$

uniformly on compact subsets of $\Omega \cup I \backslash\{0\}$.

Received by the editors December 18, 2006.

2000 Mathematics Subject Classification. Primary 30C40.

Key words and phrases. Green's function, equilibrium point, Bergman function.

This research was supported in part by NSF grant DMS-0525339. 
Proof. Since

$$
G_{\Omega}(z, w)=G_{\widehat{\Omega}}(z, w)-G_{\widehat{\Omega}}(z, \bar{w})
$$

for all $z, w \in \Omega$ such that $z \neq w,(1)$ follows from (3) after differentiation.

Let $\widehat{G}(z, w)=G_{\widehat{\Omega}}(z, w)$. Since $\widehat{G}(z, w)$ is harmonic in each variable if $z \neq w$, the function $\widehat{G}_{z}(z, w)$ has the following Taylor expansion at $w=0$ if $z \neq 0$ :

(4) $\widehat{G}_{z}(z, w)=G_{z}(z, 0)+G_{z w}(z, 0) w+G_{z \bar{w}}(z, 0) \bar{w}+$ higher powers of $w$ and $\bar{w}$.

Using (3), (4), and (1), we find that

$$
\text { (5) } \begin{aligned}
\lim _{\mathbb{H} \ni w \rightarrow 0} \frac{\frac{\partial}{\partial z} G_{\Omega}(z, w)}{\Im w} & =\lim _{w \rightarrow 0} \frac{\widehat{G}_{z}(z, w)-\widehat{G}_{z}(z, \bar{w})}{\Im w}=-2 i\left(\widehat{G}_{z \bar{w}}(z, 0)-\widehat{G}_{z w}(z, 0)\right) \\
& =\pi i\left(K_{\widehat{\Omega}}(z, 0)-L_{\widehat{\Omega}}(z, 0)\right)=\pi i K_{\Omega}(z, 0)
\end{aligned}
$$

and the limit is uniform on compact subsets of $\widehat{\Omega} \backslash\{0\}$.

Proof of Theorem 1. Using Hurwitz's theorem one can easily prove that the set $\mathbb{Z}_{\Omega}(w)$ depends continuously on $w \in \Omega$. Since $\Omega$ is open and connected and for every $w \in \Omega, \sharp\left(\mathbb{Z}_{\Omega}(w)\right)=n-1$ counting multiplicity the latter implies that $\mathbb{Z}_{\Omega}$ has at most $n-1$ connected components.

The proof of $\overline{\mathbb{Z}}_{\Omega} \subset \Omega$ is by contradiction. Suppose there is a sequence $w_{k} \in \Omega$ with $w_{k} \rightarrow w_{0}$ as $k \rightarrow \infty$ such that there exists a sequence $z_{k} \in \Omega$ with $z_{k} \rightarrow z_{0} \in$ $\partial \Omega$ such that $G_{z}\left(z_{k}, w_{k}\right)=0$. Now we consider two cases.

(1) If $w_{0} \in \Omega$, then let $z_{1}^{0}, \ldots, z_{n-1}^{0}$ be zeros of $G_{z}\left(z, w_{0}\right)$ counting multiplicity. Let $\varepsilon>0$ be sufficiently small. By Hurwitz's theorem, there is a positive integer $N$ such that for all $k \geq N$ the set $\bigcup_{j=1}^{n-1}\left\{z:\left|z-z_{j}^{0}\right|<\varepsilon\right\}$ contains exactly $n-1$ zeros of $G_{z}\left(z, w_{k}\right)$ counting multiplicity. Since $\#\left(\mathbb{Z}_{\Omega}\left(w_{k}\right)\right)=n-1$ and $z_{j}^{0} \in \Omega$ for all $j=1, \ldots, n-1$, the latter contradicts the assumption that $G_{z}\left(z, w_{k}\right)$ has a zero, say $z_{k}^{1}$, such that $z_{k}^{1} \rightarrow z_{0} \in \partial \Omega$ as $k \rightarrow \infty$.

(2) Suppose now that $w_{0} \in \partial \Omega$. Since Green's function and the Bergman kernel are conformally invariant, we may use Koebe's theorem on the conformal mapping onto a circular domain to reduce the problem to the case of domain $\Omega$ in $\mathbb{H}$ bounded by the real axis $\mathbb{R}$ and $n-1$ disjoint circles in $\mathbb{H}$. In addition, we may assume that $w_{0}=0$. Let $f_{k}(z)=G_{z}\left(z, w_{k}\right) /\left(\Im w_{k}\right)$. By equation (2) of Lemma 1, $f_{k}(z) \rightarrow K_{\Omega}(z, 0)$ uniformly on compact subsets of $\Omega$.

By a theorem of N. Suita and A. Yamada in [3], if $0 \in \partial \Omega$ the Bergman kernel $K_{\Omega}(z, 0)$ has exactly $n-1$ zeros, say $z_{1}^{0}, \ldots, z_{n-1}^{0}$, in $\Omega$ counting multiplicity. Applying Hurwitz's theorem to the sequence $f_{k}(z)$ as in $(\mathbf{1})$, we conclude that for a given sufficiently small $\varepsilon>0$ there is a positive integer $N$ such that for all $k \geq N$ the set $\bigcup_{j=1}^{n-1}\left\{z:\left|z-z_{j}^{0}\right|<\varepsilon\right\}$ contains exactly $n-1$ zeros of $f_{k}(z)$ (which coincide with zeros of $\left.G_{z}\left(z, w_{k}\right)\right)$ counting multiplicity. Since $\#\left(\mathbb{Z}_{\Omega}\left(w_{k}\right)\right)=n-1$ and $z_{j}^{0} \in \Omega$ for all $j=1, \ldots, n-1$, the latter contradicts the assumption that $G_{z}\left(z, w_{k}\right)$ has a zero, say $z_{k}^{1}$, such that $z_{k}^{1} \rightarrow z_{0} \in \partial \Omega$ as $k \rightarrow \infty$. The theorem is proved.

Remarks. (1) If $\Omega$ is infinitely connected, then $\mathbb{Z}_{\Omega}(w)=\left\{z_{k}(w)\right\}_{k=1}^{\infty}$ is infinite for every $w \in \Omega$. Since $G_{z}(z, w)$ is not constant, the uniqueness theorem for analytic functions implies that $z_{k}(w) \rightarrow \partial \Omega$ as $k \rightarrow \infty$. Thus, Theorem 1 fails for every infinitely connected domain. 
(2) We finish this note with a remark on the boundary of $\mathbb{Z}_{\Omega}$. Let $z_{0} \in \partial \mathbb{Z}_{\Omega}$. If, in addition, $z_{0} \in \mathbb{Z}_{\Omega}$, then $G_{z}\left(z_{0}, w_{0}\right)=0$ for some $w_{0} \in \Omega, z_{0} \neq w_{0}$. Let $\nabla w G_{z}(z, w)$ denote the determinant of the Jacobian matrix of $G_{z}(z, w)$ in the variable $w$. Then

$$
\nabla w G_{z}(z, w)=\frac{\pi^{2}}{4}\left(\left|L_{\Omega}(z, w)\right|^{2}-\left|K_{\Omega}(z, w)\right|^{2}\right) .
$$

By the implicit function theorem, if $\left|K_{\Omega}\left(z_{0}, w_{0}\right)\right| \neq\left|L_{\Omega}\left(z_{0}, w_{0}\right)\right|$, then $z_{0}$ is an interior point of $\mathbb{Z}_{\Omega}$. Therefore for $z_{0} \in \partial \mathbb{Z}_{\Omega}$, there are two possibilities: 1$)$ either $K_{\Omega}\left(z_{0}, w_{0}\right)=0$ for some $w_{0} \in \partial \Omega$ or 2$)\left|K_{\Omega}\left(z_{0}, w_{1}\right)\right|=\left|L_{\Omega}\left(z_{0}, w_{1}\right)\right|$ for every $w_{1} \in \Omega$ such that $G_{z}\left(z_{0}, w_{1}\right)=0$. The latter possibility seems unlikely, but we could not exclude this case.

\section{REFERENCES}

1. S. R. Bell, The Cauchy Transform, Potential Theory, and Conformal Mapping. Studies in Advanced Mathematics. CRC Press, Boca Raton, FL, 1992. MR1228442 (94k:30013)

2. A. Sebbar and Th. Falliero, Equilibrium point of Green's function for the annulus and Eisenstein series. Proc. Amer. Math. Soc. 135 (2007), 313-328. MR2255277 (2007h:30011)

3. N. Suita, A. Yamada, On the Lu Qi-Keng conjecture. Proc. Amer. Math. Soc. 59 (1976), 222-224. MR0425185 (54:13142)

Department of Mathematics and Statistics, Texas Tech University, Box 41042, LubBOCK, TEXAS 79409

E-mail address: alex.solynin@ttu.edu 